\title{
Quantum Dot Properties in a Strong Magnetic Field
}

\begin{abstract}
A. Matulis and E. Anisimovas
Semiconductor Physics Institute, Goštauto 11, 01108 Vilnius, Lithuania

We present a quasiclassical approach to few-electron quantum dots in strong magnetic fields based on the notion of a collectively rotating Wigner molecule. A quasiclassical many-particle wave function is derived and illustrated by its application to a two-electron quantum dot. In particular, we calculate the density-current correlation function (conditional current) and show that the Wigner crystal in high magnetic fields may be visualized as an ordered system of current vortices.
\end{abstract}

PACS numbers: 73.21.La, 71.10.-w, 75.75.+a

\section{Introduction}

The problem of the electronic structure of few-electron quantum dots remains in the centre of attention of solid state research [1]. Although recently substantial achievements have been made by relying on the exact numerical solution of the complicated quantum-mechanical problem, simple analytical models are also of great interest and even become increasingly more popular [3, 4]. The main interest in quantum dots is related to the electron-electron interaction and collective phenomena, such as the change of the ground state multiplicity, the electron density reconstruction, and the Wigner crystallization. The application of a strong magnetic field $B$ is known to facilitate the manifestation of the above mentioned phenomena. On the other hand, in the strong magnetic field limit one is able to construct a simplified description by means of $1 / B$ expansion.

In the present report we call attention to the manifestation of the classical nature of quantum dots in strong magnetic fields, and illustrate the application of a simple quasiclassical theory for quantum dots containing few $(2 \div 5)$ electrons. This theory is based on the assumption that the electrons are located close to their equilibrium points in a rotating classical Wigner crystal (electron ring). In this way, we construct the many-electron wave function, obtain the density-density and density-current correlators, and demonstrate that the interplay between the 
magnetic field and the Euler-liquid-like behaviour of the electron liquid gives rise to persistent currents flowing along the electron ring as well as local currents circulating around the electron density lumps.

\section{Model}

We consider a $2 \mathrm{D}$ parabolic quantum dot containing $N=2 \div 5$ electrons in a perpendicular magnetic field $\boldsymbol{B}$. The classical analysis of this system (valid in the limit $B \rightarrow \infty$ ) predicts the formation of a single ring of radius $a_{n}=a$ with electrons located equidistantly at angular positions $\alpha n(\alpha=2 \pi / n)$ as shown in Fig. 1 for the case of three electrons. It is known that a strong magnetic field forces the electron system to rotate, and in the ground state the system tries to compensate this rotation by incrementing its total angular momentum $M$. In the limit of high magnetic fields $\left(B^{-1} \ll 1\right)$ small deviations of electrons from the classical equilibrium positions (harmonic vibrations) have to be taken into account. Coupling of these vibrations to the rotation of the system as a whole complicates the construction of a quantum-mechanical description. The situation can be managed by introducing a frame rotating with a constant angular velocity $\dot{\chi}$ in which the electron system has zero total angular momentum.

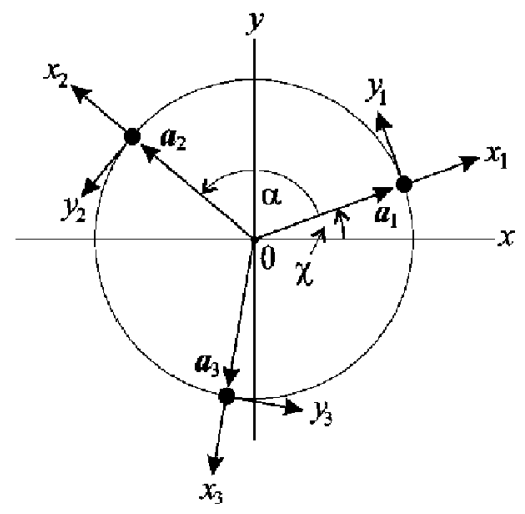

Fig. 1. Local coordinates for three electrons on a ring.

In order to derive the Hamiltonian in the rotating frame we follow Maksym [4] and start from the classical Lagrangian

$$
\begin{aligned}
& L=L_{\mathrm{mag}}-V \\
& L_{\mathrm{mag}}=\frac{1}{2} \sum_{n=1}^{N}\left(\dot{r}_{n}^{2}-\left[\boldsymbol{B} \times \boldsymbol{r}_{n}\right] \dot{r}_{n}\right),
\end{aligned}
$$




$$
V=\frac{1}{2} \sum_{n=1}^{N} \boldsymbol{r}_{n}^{2}+\sum_{\substack{n, m=1 \\ n>m}}^{N} \frac{\lambda}{\left|\boldsymbol{r}_{n}-r_{m}\right|}
$$

where $L_{\text {mag }}$ is the sum of free-electron Lagrangians in a magnetic field, and $V$ stands for the confinement and interaction energies. Here the energies are measured in units $\hbar \omega_{0}$ with $\omega_{0}$ being the characteristic frequency of the confinement potential. The coordinates are measured in units $l_{0}=\sqrt{\hbar / m^{*} \omega_{0}}$ (the oscillator lengths), and the magnetic field in units $\Phi_{0} / \pi l_{0}^{2}\left(\Phi_{0}=\pi \hbar c / e\right)$. The dimensionless Coulomb coupling constant $\lambda=l_{0} / a_{\mathrm{B}}^{*}$ is expressed as the ratio of the parabolic confinement length $l_{0}$ to the effective Bohr radius $a_{\mathrm{B}}^{*}=\epsilon \hbar^{2} / m^{*} e^{2}$.

\section{Many-electron wave function}

The details of the calculation will be published elsewhere [5], and here we merely outline the main idea which schematically can be presented as follows:

$$
L_{\mathrm{cl}, \mathrm{lab}} \rightarrow L_{\mathrm{cl}, \mathrm{rot}} \rightarrow L_{\mathrm{cl}, \mathrm{rot}, \mathrm{nm}} \rightarrow H_{\mathrm{cl}, \mathrm{rot}} \rightarrow H_{\mathrm{q}, \mathrm{rot}} \rightarrow \Psi_{\mathrm{rot}} \rightarrow \Psi_{\mathrm{lab}} \equiv \Psi .
$$

We transform the Lagrangian (1) into the rotating frame and introduce the normal modes (nm) by Fourier-transforming the local coordinates (to be used in all expressions hereafter) shown in Fig. 1. This transformation is necessary in order to deal with the additional condition (the zero total angular momentum) that sets the proper choice of the rotation velocity. The obtained Lagrangian $L_{\mathrm{cl}, \mathrm{rot}, \mathrm{nm}}$ is time independent, and the corresponding Hamiltonian is obtained through the standard replacement of velocities by the generalized momenta. Retaining only the terms of the harmonic approximation we find

$$
\begin{aligned}
H_{\mathrm{cl}, \mathrm{rot}} & =\frac{1}{2 I}\left(M+\frac{B I}{2}\right)^{2}+\frac{1}{2} U_{0}^{2} \\
+ & \frac{1}{2} \sum_{k=1}^{N-1}\left[\left(U_{k}+\frac{M v_{k}}{I_{0}}\right)^{2}+\left(V_{k}-\frac{M u_{k}}{I_{0}}\right)^{2}\right]+V .
\end{aligned}
$$

Here, the lowercase symbols $u_{k}$ and $v_{k}$ stand for the Fourier transforms of the local coordinates

$$
x_{n}=\frac{1}{N} \sum_{k=0}^{N-1} \mathrm{e}^{\mathrm{i} \alpha k(n-1)} u_{k}, \quad y_{n}=\frac{1}{N} \sum_{k=0}^{N-1} \mathrm{e}^{\mathrm{i} \alpha k(n-1)} v_{k},
$$

while the capitals $U_{k}$ and $V_{k}$ are the corresponding momenta. The symbol $M$ denotes the angular momentum, and the moment of inertia is

$$
I=\left(\sqrt{N} a+u_{0}\right)^{2}, \quad I_{0}=N a^{2} .
$$

In the harmonic approximation the quantization of the above Hamiltonian (5) and the solution of the Schödinger equation

$$
\left(H_{\mathrm{q}, \mathrm{rot}}-E\right) \Psi_{\mathrm{rot}}=0
$$


is simple. The rotation angle $\chi$ is a cyclic variable and thus the angular momentum $M$ is a constant of motion. Quantizing, we replace this momentum by the integer eigenvalue of the corresponding operator. Thus, the rotational part of the wave function is given by $\Psi^{(\text {rot })}=\exp (i M \chi)$.

Before proceeding we need to fix the radius of the ring $a$. Its value is obtained by minimizing the potential energy, namely, the first (large) and the last (small) terms in Eq. (5). We replace the moment of inertia $I$ by $I_{0}$ in the first term, and in the small last term the electron coordinates are approximated by their classical equilibrium values

$$
V_{\mathrm{eff}}(a)=\frac{1}{2 N a^{2}}\left(M+\frac{B N a^{2}}{2}\right)^{2}+N\left(\frac{1}{2} a^{2}+\frac{\lambda}{2 a} f_{N}\right) .
$$

Here, the factor $f_{N}=\sum_{n=1}^{N-1}|\sin (\alpha n / 2)|^{-1} / 2$ is the Coulomb energy per electron in a ring of unit radius. We take advantage of the different magnitude of the two contributions and carry out the minimization in two steps. Minimizing the large term we obtain a relation between the magnetic field strength and the ground state angular momentum

$$
-M=|M|=\frac{1}{2} B \mathcal{I}_{0}=\frac{1}{2} N B a^{2} .
$$

As expected, the momentum grows in absolute value with increasing magnetic field. The radius of the ring $a$ itself is obtained minimizing the smaller term of the potential with the result

$$
a=\left(\lambda f_{N} / 2\right)^{1 / 3} .
$$

Now taking into account the expansion

$$
\frac{1}{2 I}\left(M+\frac{B I}{2}\right)^{2} \approx \frac{1}{2 I_{0}}\left(M+\frac{B I_{0}}{2}\right)^{2}+\frac{1}{2} B^{2} u_{0}^{2}
$$

we see that Hamiltonian (5) is a collection of non-interacting 1D and 2D oscillators, and consequently, its ground state wave function can be represented as a product of the corresponding Gaussian factors.

Transforming the result into the laboratory frame within the harmonic accuracy we obtain the final expression for the many-electron wave function

$$
\begin{aligned}
& \Psi=\mathrm{e}^{\mathrm{i} M \chi} \mathrm{e}^{-B K / 4}, \\
& \chi=\frac{1}{N a} \sum_{n=1}^{N} y_{n}\left(1-\frac{1}{N a} \sum_{n=1}^{N} x_{n}\right),
\end{aligned}
$$




$$
K=\sum_{n=1}^{N}\left(x_{n}^{2}+y_{n}^{2}\right)+\frac{1}{N}\left[\left(\sum_{n=1}^{N} x_{n}\right)^{2}+\left(\sum_{n=1}^{N} y_{n}\right)^{2}\right]
$$

Meanwhile, Eq. (9) gives the ground-state eigenvalue $E(M, B, \lambda)=V_{\text {eff }}(a)$ with an unessential energy shift.

\section{Illustration: two electrons}

Now we illustrate the obtained many-electron wave function and the quasiclassical character of quantum dots by applying it to the simplest two-electron case. In this case the above wave function reads

$$
\begin{aligned}
\Psi= & \exp \left\{\frac{\mathrm{i} M}{2 a}\left(y_{1}+y_{2}\right)\left[1-\frac{1}{2 a}\left(x_{1}+x_{2}\right)\right]\right. \\
& \left.-\frac{B}{4}\left[3 x_{1}^{2}+2 x_{1} x_{2}+3 x_{2}^{2}+\left(y_{1}-y_{2}\right)^{2}\right]\right\} .
\end{aligned}
$$

We see that it is composed of two exponents. The first one, with an imaginary argument, is responsible for the currents in the dot while the other one, with a real argument, describes the electron density.

Although the wave function contains the complete information about the system, it is more convenient to consider simpler single-electron functions. Usually, the charge distribution is described using the single-electron density

$$
\begin{aligned}
\rho(\boldsymbol{r}) & =\int \mathrm{d}^{2} r^{\prime}\left\{\left|\Psi\left(\boldsymbol{r}, \boldsymbol{r}^{\prime}\right)\right|^{2}+\left|\Psi\left(\boldsymbol{r}^{\prime}, \boldsymbol{r}\right)\right|^{2}\right\} \\
& \sim \int \mathrm{d}^{2} r^{\prime} \exp \left\{-\frac{B}{2}\left[3 x_{1}^{2}+2 x_{1} x_{2}+3 x_{2}^{2}+\left(y_{1}-y_{2}\right)^{2}\right]\right\} \\
& \sim \exp \left\{-\frac{4 B}{3} x^{2}\right\} .
\end{aligned}
$$

We see that the electron distribution has a Gaussian profile and is centred on the classical equilibrium radius. As this distribution does not depend on the azimuthal coordinate $y$, the electron density resembles a broadened ring as shown in Fig. 2a. As expected, the ground state density retains the symmetry of the Hamiltonian.

The electron current in the dot is constructed in a similar way. For instance, the first-electron component of the many-electron current density reads

$$
j^{(1)}\left(r_{1}, r_{2}\right)=\operatorname{Im}\left\{\Psi^{*}\left(r_{1}, r_{2}\right) \nabla_{1} \Psi\left(r_{1}, r_{2}\right)\right\}+\boldsymbol{A}\left(r_{1}\right)\left|\Psi\left(r_{1}, r_{2}\right)\right|^{2},
$$

or

$$
\begin{aligned}
& j_{x}^{(1)}=\frac{B}{4}\left(y_{1}-y_{2}\right)\left|\Psi\left(r_{1}, r_{2}\right)\right|^{2} \\
& j_{y}^{(1)}=\left[\frac{1}{2 a}\left(M+B a^{2}\right)+\frac{B}{4}\left(3 x_{1}+x_{2}\right)\right]\left|\Psi\left(r_{1}, r_{2}\right)\right|^{2}
\end{aligned}
$$



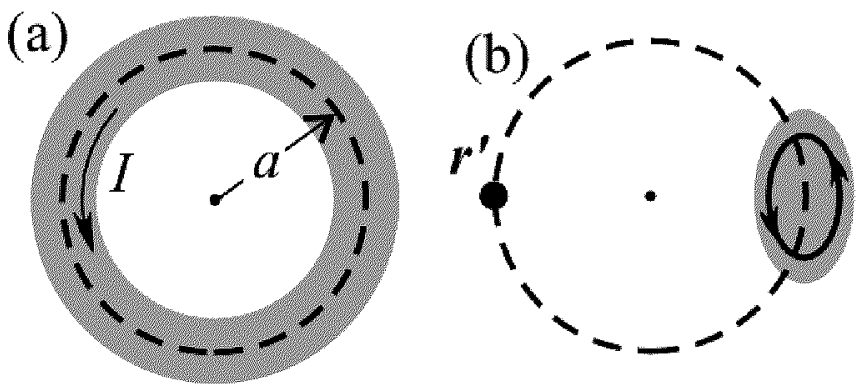

Fig. 2. Two electrons in a dot: (a) electron density (17), (b) correlation function (22) with one electron fixed at the point $r^{\prime}$.

Now, integrating the obtained current components over the second electron coordinates and keeping in mind that the contribution of both electrons is the same, the single-electron current can be presented as

$$
j_{\varphi}(\boldsymbol{r})=j_{y}(\boldsymbol{r})=2 \int \mathrm{d}^{2} r^{\prime} j_{y}^{(1)}\left(\boldsymbol{r}, \boldsymbol{r}^{\prime}\right)=\frac{1}{2 a}\left(M+B a^{2}\right) \rho(\boldsymbol{r})+\frac{2 B}{3} x \rho(\boldsymbol{r}),
$$

and $j_{x}(\boldsymbol{r})=0$. Finally, integrating the obtained expression (20) with respect to the radius we obtain the total azimuthal current flowing along the electron ring

$$
I=\int \mathrm{d} r j_{\varphi}(r) \approx \frac{1}{a} \int \mathrm{d}^{2} r j_{\varphi}(r)=\frac{1}{a^{2}}\left(M+B a^{2}\right)
$$

It seems that according to the condition (10) the azimuthal component of the current should be zero. However, this condition can be satisfied only approximately since an arbitrary magnetic field $B$ cannot be compensated exactly by a discrete value of the angular momentum $M$. Therefore, there is an azimuthal persistent current flowing in the quantum dot as indicated by an arrow in Fig. 2a, resembling the persistent currents in quantum rings [6].

However, the single-electron density (17) and current density (20) do not reveal the internal structure of the electron system. For this purpose we have to calculate the correlation functions. The simplest one is the density-density correlation function which can be obtained directly from the wave function (16) by fixing the second electron at the classical equilibrium position $x_{2}=y_{2}=0$. Thus, we obtain the following expression for the density-density correlation function:

$$
K(r)=|\Psi(r, 0)|^{2} \sim \exp \left[-\frac{B}{2}\left(3 x^{2}+y^{2}\right)\right],
$$

shown as a contour plot in Fig. 2b. It has the shape of an oval elongated in the azimuthal direction. The dimension of this electron lump is proportional to the inverse magnetic field strength $B^{-1}$.

In order to consider the currents flowing in a Wigner crystal one has to calculate the density-current correlation function (conditional current). It immediately 
follows from Eq. (19) with the second electron fixed (i.e., $x_{2}=y_{2}=0$ ). We see that this current has two components. One of them, proportional to $\left(M+B a^{2}\right)$, is related to the persistent current which was discussed above, while the other one can be presented as

$$
\boldsymbol{J}(\boldsymbol{r})=-\frac{1}{4}\left[\boldsymbol{e}_{z} \times \nabla\right] K(\boldsymbol{r})
$$

From the obtained simple expression we may conclude that the current lines are perpendicular to the gradient of the density-density correlator so that the current flows along the constant density curves as it is indicated by the solid oval with an arrow in Fig. 2b. Moreover, it is evident that

$$
\operatorname{div} \boldsymbol{J}(r)=-\frac{1}{4} \nabla\left[\boldsymbol{e}_{z} \times \nabla\right] K(r)=0,
$$

that is, in the considered quasiclassical approximation the local currents circulating around the localized electrons are conserved. Therefore, they are physically well defined even though there is no general conservation theorem for the conditional currents.

\section{Vorticity}

In order to illustrate the adequacy of the proposed quasiclassical model we compare the calculated local currents to those obtained from the exact numerical diagonalization of the Hamiltonian. It is convenient to consider the divergence div $j$

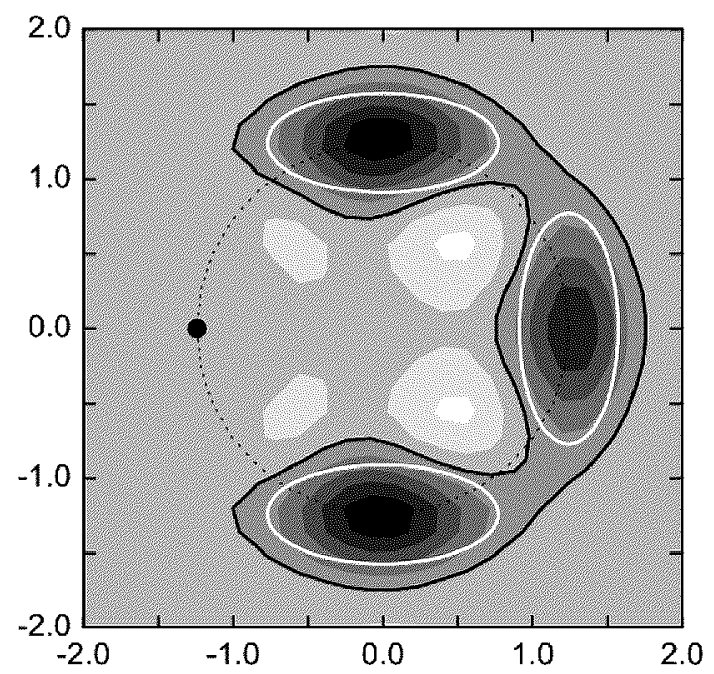

Fig. 3. Vorticity: exact diagonalization results. $N=4, \lambda=2$, and $M=-18$. The black curve indicates the zero-vorticity level, the white ovals denote the same level obtained from the quasiclassical model. 
and vorticity $\omega=\operatorname{rot}_{z} \boldsymbol{j}$ instead of the current components themselves. The exact numerical results for the vorticity in a four-electron dot are shown in Fig. 3. One of electrons is fixed at the position indicated by the black dot on the classical electron ring (marked by the dashed circle). The positive vorticity is shown by the dark areas, while the light areas indicate the negative vorticity. The closed solid curve shows the zero-vorticity level. The same zero level obtained from the quasiclassical model is indicated by the white solid oval. We note a rather good agreement, although the real vorticity distribution is broader due to the electron tunnelling between the neighbouring electron lumps which is neglected in the simplified model.

We also verified that the divergence is much smaller than the vorticity which confirms the usefulness of the proposed quasiclassical model.

In conclusion, we see that the obtained quasiclassical conditional currents are well-defined physical quantities and illustrate the formation of the Wigner crystal in quantum dots at high magnetic fields. The Wigner crystal may be visualized as an ordered system of current vortices rather than just charge density lumps.

\section{References}

[1] L.P. Kouwenhoven, D.G. Austing, S. Tarucha, Rep. Prog. Phys. 64, 701 (2001).

[2] C. Yannouleas, U. Landman, Phys. Rev. B 66, 115315 (2002); 68, 035326 (2003).

[3] E. Anisimovas, A. Matulis, M.B. Tavernier, F.M. Peeters, Phys. Rev. B 69, 075305 (2004)

[4] P.A. Maksym, Phys. Rev. B 53, 10871 (1996).

[5] E. Anisimovas, A. Matulis, F.M. Peeters, Phys. Rev. B, submitted.

[6] M. Büttiker, Y. Ymry, R. Landauer, Phys. Lett. A 96, 365 (1983). 